\title{
Atypical Bacteria and Macrolides in Asthma
}

\author{
Paraskevi Xepapadaki, MD, PhD, Ioanna Koutsoumpari, MD, Vasiliki Papaevagelou, MD, PhD, \\ Christina Karagianni, $M D$, and Nikolaos G. Papadopoulos, $M D, P h D$
}

\begin{abstract}
Chlamydophila pneumoniae and Mycoplasma pneumoniae are common pathogens causing acute illness in both the upper and lower airways. Several observations are supportive of a possible causative role of these pathogens in asthma; however, more evidence is required before this becomes meaningful in clinical practice. Atypical bacteria can enhance airway hyperresponsiveness and inflammation, both of which have been associated with exacerbations in patients with preexisting asthma. It is less clear whether the above mechanisms might also be responsible for the development of asthma. Difficulties in accurately diagnosing these infections contribute to such uncertainty. In the present report, evidence of the involvement of Chlamydophila and Mycoplasma infection in the development and the progression of asthma are reviewed.
\end{abstract}

Key words: asthma, Chlamydophila pneumoniae, Mycoplasma pneumoniae

$A$ sthma is highly prevalent worldwide and still increasing in many countries, with high morbidity, not negligible mortality, a marked effect on quality of life, and a large economic burden on society. ${ }^{1}$ Its major pathophysiologic characteristics are bronchial inflammation and airway hyperresponsiveness, which result in episodes of reversible airway obstruction. ${ }^{2}$ Among the various factors that have been involved in asthma pathogenesis, infections are prominent. Infections have been associated with triggering of acute asthma exacerbations and the initiation or persistence of asthma, but also, from a different perspective, protection from asthma initiation. ${ }^{3}$ These apparent discrepancies are probably due to the involvement of different microorganisms, intensity of infection, age, and frequency of events. ${ }^{4}$ Similarly, Chlamydophila pneumoniae (Cpn) and Mycoplasma pneumoniae $(M p n)$, agents that have been associated with atypical pneumonias and therefore colloquially termed "atypical" bacteria, have been associated with initiation, persistence, and exacerbations of asthma. ${ }^{5}$ What is of particular interest with these microbial agents is the fact that, in contrast to viruses, they are susceptible to available

Paraskevi Xepapadaki, Ioanna Koutsoumpari, Vasiliki Papaevagelou, Christina Karagianni, and Nikolaos G. Papadopoulos: Allergy Department, 2nd Pediatric Clinic, University of Athens, Athens, Greece. Correspondence to: Nikolaos G. Papadopoulos, MD, PhD, Allergy Research Center, 41 Fidippidou str, 11527 Goudi, Greece; e-mail: ngp@ allergy.gr.

DOI 10.2310/7480.2008.00012 antimicrobial therapy. Herein we review evidence of the role of atypical bacteria in asthma pathogenesis and the potential role of macrolide antibiotics in this respect.

\section{Atypical Bacteria and Their Associations with Asthma}

$C p n$ is a relatively "new" respiratory pathogen, first discovered in 1986, characterized by its propensity to cause chronic, unapparent infections. Serologic studies indicate that it is considerably prevalent worldwide. ${ }^{6}$ Host immunity elicited by $C p n$ infection appears to be shortlived and only partial, and recurrent infections are therefore common. Furthermore, Cpn posttreatment persistence rates range from 13 to $56 \%{ }^{7} \mathrm{Mpn}$ is both an intracellular and an extracellular pathogen that primarily infects ciliated epithelial cells and alveolar macrophages. ${ }^{8,9}$ It is very common for $M p n$ to be isolated from sputum or the upper respiratory tract for several weeks to several months after recovery from clinical illness. ${ }^{10}$

These agents infect the human bronchial tree, causing ciliary dysfunction and epithelial damage and disturbing airway physiology through a number of different pathways. Cpn and $M p n$ species are able to generate proinflammatory cytokines both in vivo and in vitro. ${ }^{11}$ Both agents are involved in a number of respiratory diseases, including upper respiratory tract illnesses such as rhinitis, pharyngitis, and otitis, as well as atypical pneumonia and bronchitis, lung cancer, and, recently, chronic obstructive pulmonary disease. ${ }^{12-14}$ 
Atypical bacteria have been associated with airway dysfunction in a number of ways. Infection with these agents can induce transient wheezing in individuals with no history of asthma. ${ }^{15,16}$ It has also been observed that infection with Mpn can produce long-term impairment in airway function, even in asymptomatic children. ${ }^{17}$ Atypical bacteria, mostly $M p n$, have been associated with acute exacerbations of asthma in children and adults. ${ }^{18,19}$ In some cases, atypical bacterial infection has preceded the onset of asthma, especially in adults, ${ }^{20,21}$ and, more recently, has also been suggested in children. ${ }^{22}$

Many studies have shown a correlation between the presence of antibodies to atypical bacteria and chronic asthma, leading investigators to suggest a role for these agents in the persistence of asthma or resistance to steroids. ${ }^{23}$ Alternatively, it is possible that chronic asthmatic patients may be more susceptible to such infections.

However, other studies have observed no differences in $C p n$-specific antibodies between new asthma cases, established asthma and healthy controls. ${ }^{24-26}$

\section{Atypical Bacteria Inducing Wheezing and/or Asthma Exacerbations}

It has been estimated that most people may have three or more Cpn infections during their lifetime. Available evidence supports a role for acute $C p n$ and $M p n$ respiratory tract infections as a trigger for 5 to $30 \%$ of wheezing episodes and/or asthma exacerbations. ${ }^{27}$

$C p n$ and $M p n$ infections may also be accompanied by wheezing in children considered not to have asthma. ${ }^{28,29}$ The incidence of wheezing in acute Mpn infection was reported to be 25 to $40 \%$ in the pediatric population. ${ }^{16}$

Most of the studies that investigated the role of $C p n$ and Mpn in acute asthma symptoms were indicative of a positive association. ${ }^{19-21,23,30-36} \mathrm{Hahn}$ and colleagues were the first to identify a consistent association between wheezing episodes and anti-Cpn titers in patients with acute lower respiratory illness. ${ }^{20}$ They reported that the proportion of patients with antibodies to $C p n$ was considerably higher in those with wheezing than those without (33\% vs $17 \%$, respectively). ${ }^{20}$ The same authors further extended their observations, thus showing seroreactivity for $C p n$ in $80 \%$ of patients with acute asthmatic bronchitis compared with $53 \%$ of nonwheezing control subjects in a dose-response manner. ${ }^{21}$ Infection with $C p n$ has been associated with $10 \%$ of acute exacerbations in adult asthmatics. ${ }^{19}$ In accordance, anti-C heat shock protein IgG antibodies were significantly associated with exacerbations but only in asthmatic adult subjects $(p<.0001) .^{30}$ However, in one of the largest studies, undertaken in 100 adults with acute asthma exacerbations who were compared with nonasthmatic matched control subjects and in which immunofluorescence or enzyme immunoassay methods were used to establish a serologic diagnosis, only Mpn was associated with hospitalization for asthma exacerbation (18 vs $3 \%, p=.0006$ in asthmatics and controls, respectively). No significant differences in the rates of acute infection with $C p n$, Legionella spp, or Coxiella were noted between the two groups. ${ }^{37}$ Moreover, when reverse transciptase-polymerase chain reaction (PCR) methods were used in patients hospitalized with acute asthma and patients with stable asthma, no atypical bacteria infection was detected, further contributing to the controversial role of atypical bacteria in acute asthma. ${ }^{33}$ Other studies have also failed to establish an association between infection with Cpn or Mpn and severe asthma exacerbations. ${ }^{35,38}$

Studies have also been performed in children, with the majority showing significant correlations between infection with atypical bacteria and exacerbation of asthma symptoms. One of the first studies, performed in more than 100 children, indicated that Cpn infection, in terms of positive cultures or serologic titers, was able to trigger acute episodes of wheezing in children with asthma. ${ }^{33}$ Moreover, the study found that treatment of Cpn infection may improve the course of "reactive airway disease." Cunningham and colleagues studied 108 children for 13 months. They showed that immune responses to $C p n$ detected in 292 nasal samples taken during symptomatic episodes were positively associated with the frequency of asthma exacerbations. ${ }^{32}$ Moreover, this group of children maintained a longer PCR positivity for $C p n$, suggestive of a chronic infection. Other studies have also shown that children with wheezing have a significantly higher incidence of acute infection with $C p n$ and $M p n$ than healthy controls $\left(p=.01\right.$ for each pathogen). ${ }^{39}$ The same authors suggested a potential role for interleukin (IL)-5 as a link between Mpn infection and acute wheezing by showing higher levels of IL-5 in wheezing children with Mpn infection than in those with asymptomatic acute infection without wheeze. ${ }^{34}$ However when PCR methods were used, infection with Cpn and Mpn was identified only in 4.5 and $2.2 \%$, respectively, of children hospitalized with severe asthma symptoms. ${ }^{40}$ More recently, Biscardi and collegues found that Mpn was present in $20 \%$ of exacerbations in asthmatic children requiring hospitalization, whereas $50 \%$ of children experiencing their first asthmatic attack were also positive for $M p n^{22}$ 
The high proportion of studies that have reported a link between acute exacerbations of asthma and atypical bacteria infection suggests that these pathogens may play a significant role in such exacerbations. There are still unresolved issues both with respect to microorganism identification and study design, before an etiologic association can be firmly established.

\section{Atypical Bacteria at the Beginning of Asthma}

Several studies have assessed the role of Cpn and Mpn infection in the initiation of asthma symptoms, unfortunately with conflicting findings. More than 15 years ago, Hahn and colleagues reported that significantly more patients with serologic evidence of Cpn infection developed asthmatic bronchitis in a dose-response manner. ${ }^{20}$ Interestingly, in a cross-sectional study, subjects with lateonset nonatopic asthma and the presence of $\operatorname{IgG}$ antibodies to $C p n$ showed a significant decline in postbronchodilator forced expiratory volume in 1 second $(\mathrm{FEV})_{1} /$ forced vital capacity compared with subjects without evidence of $C p n$ infection. ${ }^{41}$ In supporting the previous data, experimental $M p n$ infection in a murine model resulted in chronic pulmonary disease characterized by airway hyperreactivity, obstruction, and histologic inflammation. ${ }^{15}$ However, the aforementioned associations between CPn infection and the increased risk of presenting new asthma symptoms could not be verified in other studies. ${ }^{42}$

Studies conducted in children have also provided inconsistent data regarding the relationship between atypical bacteria infection and the beginning of asthma. In a group of 119 children hospitalized for acute asthma, $62 \%$ of first-time asthmatics with seropositivity for either $C p n$ or $M p n$ infection experienced recurrent asthma episodes, whereas only $27 \%$ of pathogen-free patients had a subsequent exacerbation, indicating a significant role for these pathogens for subsequent asthma activity. ${ }^{22}$ In contrast, other studies failed to verify such associations between positive Cpn serology and newly diagnosed asthma cases, regardless of the serologic method used, ${ }^{24}$ whereas even a possible protective role of $C p n$ upper respiratory infection, in preschool- and school-aged children, for childhood asthma has been suggested. ${ }^{43}$

In conclusion, evidence of a causal role of Cpn or $M p n$ in the initiation of asthma is inconsistent. Moreover, it should be noted that a wheezing episode associated with a $C p n$ and/or Mpn infection does not necessarily predict the initiation of a chronic inflammatory response of the airways, such as asthma.

\section{Atypical Bacteria in Chronic Stable Asthma}

Several studies have addressed the hypothesis that Cpn and $M p n$ are implicated in the course of chronic stable asthma, with the majority (15 of 19 studies) supporting a positive correlation between infection with these pathogens and asthma perpetuation. ${ }^{44}$ Black and colleagues examined in the context of a large multinational study the possibility that chronic Cpn infection may lead to increased asthma severity. ${ }^{45}$ A positive association was found between antibodies to CPn and the use of high-dose inhaled steroids and higher daytime symptom scores. It has also been shown in adults with serologic markers indicative of persistent $C p n$ infection that asthma was significantly more common compared with normal controls. ${ }^{46}$ In a bronchoscopy study, an association between $C p n$ and $M p n$, measured by means of PCR, and stable chronic asthma was sought; $56 \%$ of asthmatic patients had a positive PCR for $C p n(n=6)$ or Mpn $(n=25)$ compared with only one control subject. ${ }^{25}$ In accordance, colonization and/or infection with $M p n$ have been shown to be significantly more frequent in patients with asthma (10 of 18) than nonasthmatic controls ( 1 of $11, p=.02$ ). ${ }^{47}$ To further support a causal relationship, studies have established a dose-response relationship between the presence of atypical bacteria and asthma. In specific, heat shock protein 60 IgA antibodies and other serology markers related to $C p n$ infection were inversely associated with pulmonary function, as measured by $\mathrm{FEV}_{1}$ and accordingly associated with a higher daytime symptom score and other markers of asthma severity. ${ }^{45,48}$ The timing of the infection may also play an important role in the induction of allergic responses. The presence of $M p n$ infection after allergen sensitization and challenge can increase $\mathrm{T}$ helper 2 inflammation and enhance mucin 5 (MUC5) gene expression and airway hyperresponsiveness in a murine model. ${ }^{49}$ The same investigators also demonstrated increased collagen deposition and elevated levels of transforming growth factor $\beta_{1}$ in animal models that are allergen challenged and subsequently infected with $M p n{ }^{50}$

To what extent the atopic status may influence Cpn and $M p n$ infection is still uncertain. One controlled study in 332 asthmatic subjects suggested that nonatopic individuals presented higher levels of IgG antibodies to $C p n$ (odds ratio 6.0), ${ }^{14}$ whereas, more recently, Ferrari and colleagues showed that serologic evidence of $C p n$ infection was positively associated with cough and phlegm and possibly involved in the pathogenesis of chronic respiratory infection in atopic children. ${ }^{51}$ However, in a recent study including 104 pediatric patients with newly diag- 
nosed asthma versus 120 matched healthy controls, no differences were found with respect to $C p n$-specific antibodies regardless of the detection method used. ${ }^{24}$

The aforementioned evidence is supportive of a significant correlation between Cpn and Mpn and chronic stable asthma. Nevertheless, whether such associations imply a causative role or an increased susceptibility of asthmatic patients to colonization with atypical bacteria cannot be concluded from the current evidence.

\section{Macrolide Antibiotics}

Therapy for acute asthma has remained relatively unchanged for several years. Macrolides are not currently included among the recommended treatments for either acute or chronic asthma, although the initial experience with macrolides in treating asthma dates back more than 40 years. Macrolide antibiotics have been suspected of having beneficial effects in patients with asthma since the 1950s. ${ }^{52}$ Several studies have shown benefits, without, however, being conclusive enough to reach recommendations for clinical practice. ${ }^{53}$ It has been suggested that macrolides may act on asthma as both antimicrobials against $M p n$ and CPn and anti-inflammatory agents, a function confirmed in several studies. ${ }^{54}$

Ketolides are a new class of antibacterial agents, very closely related to macrolides, showing sufficient accumulation in macrophages, epithelial cells, and neutrophils, making them suitable for the treatment of infections caused by intracellular pathogens. ${ }^{44}$

\section{Immunomodulatory and Anti-inflammatory Effects of Macrolides}

Immunomodulation has been defined as suppressing hyperimmunity and inflammation without overt immunosuppression. Macrolides have been shown to decrease bacterial adherence and virulence, biofilm formation, and mucus hypersecretion. Only 14- and 15-membered macrolides possess anti-inflammatory properties that may contribute to the clinical benefits observed in patients with airway inflammation. ${ }^{55}$ They can modulate the function of several inflammatory cells, such as polymorphonuclear leukocytes, lymphocytes, and macrophages, although they seem to affect neutrophil migration, the oxidative burst in phagocytes, the production of proinflammatory mediators (eg, tumor necrosis factor $\alpha$, IL-8, IL-6, and IL-1 $\beta$ ), and eosinophilic inflammation. ${ }^{56}$ The most important molecular targets in asthma appear to be the transcription factors activator protein 1 and nuclear factor $\kappa \mathrm{B}$. There is also evidence that macrolides may affect corticosteroid metabolism, thus increasing their activity (see below).

In contradistinction, macrolides can acutely enhance host defense by the production of inflammatory mediators such as IL-1 by macrophages and IL-2 by lymphoid cells. ${ }^{57}$

\section{Clinical Trials of Macrolide Therapy in Asthma}

Several studies have been conducted to evaluate the effect of macrolides on asthmatic patients; however, only a few are well controlled. Several years ago, Hahn was the first to suggest that antichlamydial treatment could affect the natural history of asthma in seroactive patients with $C p n$ by means of improvement in pulmonary function measurements and asthma symptoms. ${ }^{58}$ Clarithromycin has been shown to decrease methylprednisolone clearance and increase plasma methylprednisolone levels, probably resulting in reduced bronchial hyperresponsiveness in adults and children with asthma. ${ }^{59,60}$ Moreover, clarithromycin suppresses bronchial hyperresponsiveness associated with eosinophilic inflammation and potentiates glucocorticoid responsiveness in asthmatics. ${ }^{61,62}$

To assess the role of antibiotics in the treatment of persistent asthma, the Chlamydia pneumoniae, Asthma Roxithromycin multinational study, a randomized, double-blind, placebo-controlled trial, was conducted in adult asthmatic subjects with serologic evidence of $C p n$ infection. ${ }^{63}$ The authors showed that 6 weeks of treatment with roxithromycin resulted in significant improvement in lung function; nevertheless, there was no improvement in asthma symptoms. In the same context, Kraft and colleagues demonstrated that clarithromycin, administered for 6 weeks in adult asthmatics, elicited an increase in $\mathrm{FEV}_{1}$ and a decrease in the bronchial expression of IL-5, but only in patients with PCR-documented atypical infection. ${ }^{64}$ Overall, a systematic review of macrolide use on stable chronic asthma showed a positive effect on clinical symptoms and markers of eosinophilic inflammation but concluded that further studies will be required for confirmation. ${ }^{65}$

With respect to acute asthma, a recent multicenter, double-blind, randomized, placebo-controlled study assessed the efficacy of oral telithromycin for 10 days as a supplement to standard treatment for patients with an acute asthma exacerbation. Patients in the treatment group showed significant improvements in both symptoms and lung function, suggesting that this treatment may provide some benefit. The effect did not seem to crucially depend on the presence of atypical bacteria, although this was not conclusive. ${ }^{66}$ Therefore, further workup is essential to 
identify patient groups most likely to benefit from new treatment approaches in asthma exacerbations. ${ }^{67}$

Studies in children have also shown positive effects of antichlamydial treatment in asthma-related symptoms. Clarithromycin administration significantly reduced the duration and the risk of subsequent wheezing episodes in children with evidence of $M p n$ and Cpn infection compared with placebo. ${ }^{68}$

\section{Conclusions}

There is increasing evidence that infection with atypical pathogens may play a important role in the induction of asthma symptoms in the context of chronic persistent asthma or asthma exacerbations. Whether Cpn and $M p n$ infection or airway localization indicate an increased susceptibility to infection leading to increased frequency of detection or whether these organisms play a more direct role in the pathogenesis of asthma, acutely or chronically, is not fully resolved. There is a clear requirement for standardization in detection methods for atypical pathogens. In addition, well-controlled research is urgently required to further estimate the clinical benefit of antibiotic treatment in asthmatic patients.

\section{References}

1. Asher MI, Montefort S, Bjorksten B, et al. Worldwide time trends in the prevalence of symptoms of asthma, allergic rhinoconjunctivitis, and eczema in childhood: ISAAC Phases One and Three repeat multicountry cross-sectional surveys. Lancet 2006;368:73343.

2. Bousquet J, Clark TJ, Hurd S, et al. GINA guidelines on asthma and beyond. Allergy 2007;62:102-12.

3. Martinez FD. Genes, environments, development and asthma: a reappraisal. Eur Respir J 2007;29:179-84.

4. Xepapadaki P, Papadopoulos NG. Viral infections and allergies. Immunobiology 2007;212:453-9.

5. Nisar N, Guleria R, Kumar S, et al. Mycoplasma pneumoniae and its role in asthma. Postgrad Med J 2007;83:100-4.

6. Grayston JT, Kuo CC, Wang SP, Altman J. A new Chlamydia psittaci strain, TWAR, isolated in acute respiratory tract infections. N Engl J Med 1986;315:161-8.

7. Harris JA, Kolokathis A, Campbell M, et al. Safety and efficacy of azithromycin in the treatment of community-acquired pneumonia in children. Pediatr Infect Dis J 1998;17:865-71.

8. Peeling RW, Brunham RC. Chlamydiae as pathogens: new species and new issues. Emerg Infect Dis 1996;2:307-19.

9. Hammerschlag MR. The intracellular life of chlamydiae. Semin Pediatr Infect Dis 2002;13:239-48.

10. Blasi F. Atypical pathogens and respiratory tract infections. Eur Respir J 2004;24:171-81.

11. Martin RJ. Infections and asthma. Clin Chest Med 2006;27:87-98, vi.
12. Littman AJ, Jackson LA, Vaughan TL. Chlamydia pneumoniae and lung cancer: epidemiologic evidence. Cancer Epidemiol Biomarkers Prev 2005;14:773-8.

13. Tsolia MN, Psarras S, Bossios A, et al. Etiology of communityacquired pneumonia in hospitalized school-age children: evidence for high prevalence of viral infections. Clin Infect Dis 2004;39:6816.

14. von Hertzen $\mathrm{L}$, Isoaho $\mathrm{R}$, Leinonen $\mathrm{M}$, et al. Chlamydia pneumoniae antibodies in chronic obstructive pulmonary disease. Int J Epidemiol 1996;25:658-64.

15. Hardy RD, Jafri HS, Olsen $\mathrm{K}$, et al. Mycoplasma pneumoniae induces chronic respiratory infection, airway hyperreactivity, and pulmonary inflammation: a murine model of infection-associated chronic reactive airway disease. Infect Immun 2002;70:649-54.

16. Principi N, Esposito S. Mycoplasma pneumoniae and Chlamydia pneumoniae cause lower respiratory tract disease in paediatric patients. Curr Opin Infect Dis 2002;15:295-300.

17. Katz B, Waites K. Emerging intracellular bacterial infections. Clin Lab Med 2004;24:627-49, vi.

18. Johnston SL, Pattemore PK, Sanderson G, et al. Community study of role of viral infections in exacerbations of asthma in 9-11 year old children. BMJ 1995;310:1225-9.

19. Allegra L, Blasi F, Centanni S, et al. Acute exacerbations of asthma in adults: role of Chlamydia pneumoniae infection. Eur Respir J 1994;7:2165-8.

20. Hahn DL, Dodge RW, Golubjatnikov R. Association of Chlamydia pneumoniae (strain TWAR) infection with wheezing, asthmatic bronchitis, and adult-onset asthma. JAMA 1991;266:225-30.

21. Hahn DL, Golubjatnikov R. Asthma and chlamydial infection: a case series. J Fam Pract 1994;38:589-95.

22. Biscardi S, Lorrot M, Marc E, et al. Mycoplasma pneumoniae and asthma in children. Clin Infect Dis 2004;38:1341-6.

23. Thumerelle C, Deschildre A, Bouquillon C, et al. Role of viruses and atypical bacteria in exacerbations of asthma in hospitalized children: a prospective study in the Nord-Pas de Calais region (France). Pediatr Pulmonol 2003;35:75-82.

24. Korppi M, Paldanius M, Hyvarinen A, et al. Chlamydia pneumoniae and newly diagnosed asthma: a case-control study in 1 to 6-year-old children. Respirology 2004;9:255-9.

25. Martin RJ, Kraft M, Chu HW, et al. A link between chronic asthma and chronic infection. J Allergy Clin Immunol 2001;107:595-601.

26. Tuuminen T, Edelstein I, Punin A, et al. Use of quantitative and objective enzyme immunoassays to investigate the possible association between Chlamydia pneumoniae and Mycoplasma pneumoniae antibodies and asthma. Clin Microbiol Infect 2004;10: $345-8$.

27. Gern JE, Lemanske RF Jr. Infectious triggers of pediatric asthma. Pediatr Clin North Am 2003;50:555-75, vi.

28. Gendrel D, Raymond J, Moulin F, et al. Etiology and response to antibiotic therapy of community-acquired pneumonia in French children. Eur J Clin Microbiol Infect Dis 1997;16:388-91.

29. Principi N, Esposito S, Blasi F, Allegra L. Role of Mycoplasma pneumoniae and Chlamydia pneumoniae in children with community-acquired lower respiratory tract infections. Clin Infect Dis 2001;32:1281-9.

30. Betsou F, Sueur JM, Orfila J. Anti-Chlamydia pneumoniae heat shock protein 10 antibodies in asthmatic adults. FEMS Immunol Med Microbiol 2003;35:107-11. 
31. Cook PJ, Davies P, Tunnicliffe W, et al. Chlamydia pneumoniae and asthma. Thorax 1998;53:254-9.

32. Cunningham AF, Johnston SL, Julious SA, et al. Chronic Chlamydia pneumoniae infection and asthma exacerbations in children. Eur Respir J 1998;11:345-9.

33. Emre U, Roblin PM, Gelling $\mathrm{M}$, et al. The association of Chlamydia pneumoniae infection and reactive airway disease in children. Arch Pediatr Adolesc Med 1994;148:727-32.

34. Esposito S, Droghetti R, Bosis S, et al. Cytokine secretion in children with acute Mycoplasma pneumoniae infection and wheeze. Pediatr Pulmonol 2002;34:122-7.

35. Meloni F, Paschetto E, Mangiarotti P, et al. Acute Chlamydia pneumoniae and Mycoplasma pneumoniae infections in community-acquired pneumonia and exacerbations of COPD or asthma: therapeutic considerations. J Chemother 2004;16:70-6.

36. Miyashita N, Kubota Y, Nakajima M, et al. Chlamydia pneumoniae and exacerbations of asthma in adults. Ann Allergy Asthma Immunol 1998;80:405-9.

37. Lieberman D, Printz S, Ben-Yaakov M, et al. Atypical pathogen infection in adults with acute exacerbation of bronchial asthma. Am J Respir Crit Care Med 2003;167:406-10.

38. Green RM, Custovic A, Sanderson G, et al. Synergism between allergens and viruses and risk of hospital admission with asthma: case-control study. BMJ 2002;324:763.

39. Esposito S, Blasi F, Arosio C, et al. Importance of acute Mycoplasma pneumoniae and Chlamydia pneumoniae infections in children with wheezing. Eur Respir J 2000;16:1142-6.

40. Freymuth F, Vabret A, Brouard J, et al. Detection of viral, Chlamydia pneumoniae and Mycoplasma pneumoniae infections in exacerbations of asthma in children. J Clin Virol 1999;13:131-9.

41. ten Brinke A, van Dissel JT, Sterk PJ, et al. Persistent airflow limitation in adult-onset nonatopic asthma is associated with serologic evidence of Chlamydia pneumoniae infection. J Allergy Clin Immunol 2001;107:449-54.

42. Pasternack $\mathrm{R}$, Huhtala $\mathrm{H}$, Karjalainen J. Chlamydophila (Chlamydia) pneumoniae serology and asthma in adults: a longitudinal analysis. J Allergy Clin Immunol 2005;116:1123-8.

43. Schmidt SM, Muller CE, Wiersbitzky SK. Inverse association between Chlamydia pneumoniae respiratory tract infection and initiation of asthma or allergic rhinitis in children. Pediatr Allergy Immunol 2005;16:137-44.

44. Johnston SL, Martin RJ. Chlamydophila pneumoniae and Mycoplasma pneumoniae: a role in asthma pathogenesis? Am J Respir Crit Care Med 2005;172:1078-89.

45. Black PN, Scicchitano R, Jenkins CR, et al. Serological evidence of infection with Chlamydia pneumoniae is related to the severity of asthma. Eur Respir J 2000;15:254-9.

46. Falck G, Gnarpe J, Hansson LO, et al. Comparison of individuals with and without specific IgA antibodies to Chlamydia pneumoniae: respiratory morbidity and the metabolic syndrome. Chest 2002;122:1587-93.

47. Kraft M, Cassell GH, Henson JE, et al. Detection of Mycoplasma pneumoniae in the airways of adults with chronic asthma. Am J Respir Crit Care Med 1998;158:998-1001.

48. Huittinen T, Hahn D, Anttila T, et al. Host immune response to Chlamydia pneumoniae heat shock protein 60 is associated with asthma. Eur Respir J 2001;17:1078-82.
49. Chu HW, Honour JM, Rawlinson CA, et al. Effects of respiratory Mycoplasma pneumoniae infection on allergen-induced bronchial hyperresponsiveness and lung inflammation in mice. Infect Immun 2003;71:1520-6.

50. Chu HW, Rino JG, Wexler RB, et al. Mycoplasma pneumoniae infection increases airway collagen deposition in a murine model of allergic airway inflammation. Am J Physiol Lung Cell Mol Physiol 2005;289:L125-33.

51. Ferrari M, Poli A, Olivieri M, et al. Respiratory symptoms, asthma, atopy and Chlamydia pneumoniae IgG antibodies in a general population sample of young adults. Infection 2002;30:203-7.

52. Kaplan MA, Goldin M. The use of triacetyloleandomycin in chronic infectious asthma. Antibiot Annu 1958;6:273-6.

53. Hirschmann JV. Antibiotics for common respiratory tract infections in adults. Arch Intern Med 2002;162:256-64.

54. Blasi F, Johnston SL. The role of antibiotics in asthma. Int J Antimicrob Agents 2007;29:485-93.

55. Shinkai M, Rubin BK. Macrolides and airway inflammation in children. Paediatr Respir Rev 2005;6:227-35.

56. Gotfried MH. Macrolides for the treatment of chronic sinusitis, asthma, and COPD. Chest 2004;125:52S-60S; quiz S-1S.

57. Shinkai M, Foster GH, Rubin BK. Macrolide antibiotics modulate ERK phosphorylation and IL-8 and GM-CSF production by human bronchial epithelial cells. Am J Physiol Lung Cell Mol Physiol 2006;290:L75-85.

58. Hahn DL. Treatment of Chlamydia pneumoniae infection in adult asthma: a before-after trial. J Fam Pract 1995;41:345-51.

59. Kostadima E, Tsiodras S, Alexopoulos EI, et al. Clarithromycin reduces the severity of bronchial hyperresponsiveness in patients with asthma. Eur Respir J 2004;23:714-7.

60. Fost DA, Leung DY, Martin RJ, et al. Inhibition of methylprednisolone elimination in the presence of clarithromycin therapy. J Allergy Clin Immunol 1999;103:1031-5.

61. Amayasu H, Yoshida S, Ebana S, et al. Clarithromycin suppresses bronchial hyperresponsiveness associated with eosinophilic inflammation in patients with asthma. Ann Allergy Asthma Immunol 2000;84:594-8.

62. Spahn JD, Fost DA, Covar R, et al. Clarithromycin potentiates glucocorticoid responsiveness in patients with asthma: results of a pilot study. Ann Allergy Asthma Immunol 2001;87:501-5.

63. Black PN, Blasi F, Jenkins CR, et al. Trial of roxithromycin in subjects with asthma and serological evidence of infection with Chlamydia pneumoniae. Am J Respir Crit Care Med 2001;164: 536-41.

64. Kraft M, Cassell GH, Pak J, Martin RJ. Mycoplasma pneumoniae and Chlamydia pneumoniae in asthma: effect of clarithromycin. Chest 2002;121:1782-8.

65. Richeldi L, Ferrara G, Fabbri LM, et al. Macrolides for chronic asthma. Cochrane Database Syst Rev 2005;4:CD002997.

66. Johnston SL, Blasi F, Black PN, et al. The effect of telithromycin in acute exacerbations of asthma. N Engl J Med 2006;354:1589-600.

67. Little FF. Treating acute asthma with antibiotics—not quite yet. N Engl J Med 2006;354:1632-4.

68. Esposito S, Blasi F, Bosis F, et al. Efficacy of clarithromycin for the treatment of acute episodes of bronchospasm in children with a history of recurrent wheezing. 22nd annual meeting of European Society for Pediatric Infectious Diseases (ESPID). Tampere, Finland, 2004. 